\title{
Exploratory Designing a Magnetic Induction Tomography Sensor Coil Circuit for Agarwood
}

\author{
Nurfarahin Ishak, Chua King Lee, Siti Zarina Mohd Muji, Abdul Azlin Abdul Latip
}

\begin{abstract}
Magnetic induction tomography (MIT) is an imaging modality focused on tracing the transmission of electrical conductivity within the body. This technique used to image electromagnetic properties of an object by using the eddy current effect. This paper explains the primary analog transceiver circuit of MIT. This is a surrogate design of the analog system in the electronic components for pattern recognition and conditioning. This MIT system operating with a single excitation signal frequency at $10 \mathrm{MHz}$. The input voltage received by the receiver sensor would become the circuit input which contained information. The four stages process in the receiver circuit successfully captured the signal from the transmitter. These subsystems have their functions and can be put into effect in many ways. Therefore, the circuit was used to be reliable at agarwood samples. The approach transceiver circuit were successful and functional for MIT coil sensing. The input voltage feedback depending on the conductivity of the samples. As the dielectric properties of samples are high, the input voltage at the receiver also high. Therefore, $10 \mathrm{MHz}$ can use for agriculture while this range of frequency is usually used in biomedical applications. Series - parallel circuit gives a greater induction factor and therefore more induced voltage for the load of the receiver.
\end{abstract}

Keywords: Magnetic induction tomography, excitation circuit, receiver circuit, coils

\section{INTRODUCTION}

$\mathrm{M}$ Magnetic induction tomography (MIT) direct link sensing coils for mapping the electromagnetic position of the materials. As this is a non-destructive and contactless method, it has widely used in biomedical imaging[1][2]. MIT is an imaging modality designed to rebuild the internal conductivity distribution of the body [3]. MIT also used the internal distribution of passive electromagnetic of the object. It consists of a transceiver where the objects are tested located at the center of the system. The coils are used as transmitters and receivers. The fundamental MIT system can be

Manuscript received on January 18, 2021.

Revised Manuscript received on February 26, 2021.

Manuscript published on February 28, 2021

* Correspondence Author

Nurfarahin Ishak*, Faculty of Electrical and Electronics Engineering, Universiti Tun Hussein Onn Malaysia, Johor, Malaysia. Email: farah.ishak25@gmail.com

Chua King Lee, Faculty of Electrical and Electronics Engineering, Universiti Tun Hussein Onn Malaysia, Johor, Malaysia. Email: chua.uthm.edu.my

Siti Zarina Mohd Muji, Faculty of Electrical and Electronics Engineering, Universiti Tun Hussein Onn Malaysia, Johor, Malaysia. Email: szarina.uthm.edu.my

Abdul Azlin Bin Abdul Latip, Smart Modular Technologies (M) Sdn. Bhd, Pulau Pinang, Malaysia, Email: the icare@yahoo.com

(c) The Authors. Published by Blue Eyes Intelligence Engineering and Sciences Publication (BEIESP). This is an open access article under the CC BY-NC-ND license (http://creativecommons.org/licenses/by-nc-nd/4.0/) demonstrated via using eddy current theories as in Figure 1 [4][5][6]. Concisely, the primary field was generated by the transmitter using the AC source and penetrate to the objects. The eddy currents are induced within the object because of the conductivity properties of the magnetic field. Then, these eddy currents generate the second field which contains the information on conductivity distributions. The induced voltage can be measured from this electrical field [7]. This paper discusses a transceiver designed for use in the MIT agriculture prototype system. In principle, electromagnetic induction tomography is similar to other electrical tomography mechanisms and complements. For example, electrical resistance tomography (ERT) and electrical capacitance tomography (ECT) in the effective realization of a set of methods based on electrical impedance measurement (either resistance, capacitance or inductance).

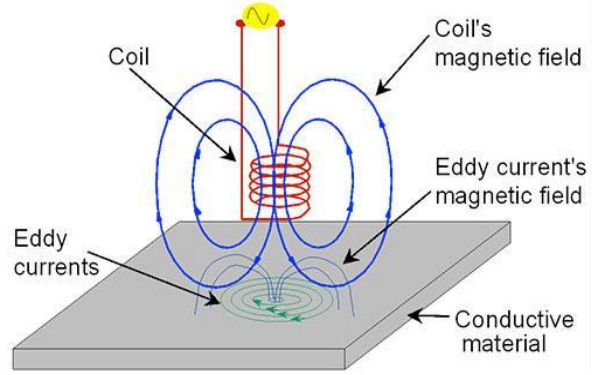

Figure 1: The eddy current principle[4]

All tomographic techniques are used the electric field excitation. For example, Figure 2 shows how MIT operates in an exciting field of focus with a magnetic field. To develop the magnetic field within the object field, the sinusoidal current is passed through coil 1.

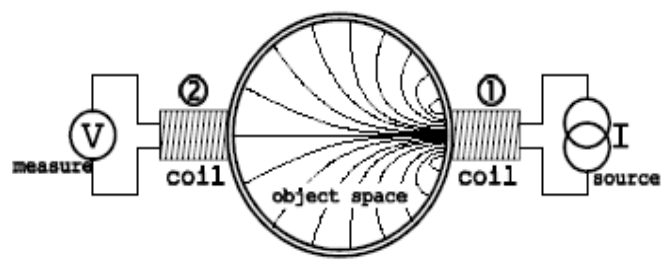

Figure 2: Example of MIT coils concept[1]

When the object field is empty (filled with air), assume a background condition with a relative permeability is assumed to equal to one. A significant voltage is induced across the coil in this condition. Coil 2 corresponds to the measurement of the background or space. The temporal variation of the magnetic field and thus the coupling between the coils is changed by the introduction of magnetic (relative permeability > 1) and/or metallic (high conductivity) elements within the space of the object. Generally,

Published By:

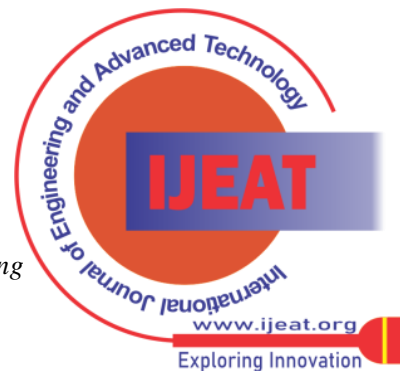


the magnetic field improves the coupling between the coils leading to increased measurements, although the objects are usually attenuated by losses of eddy current in the material itself.[1][8]. Next, the MIT circuit was tested at the agarwood samples. The samples were contained agarwood formation and without agarwood formation. Each sample has different relative permittivity and conductivity measurements to enable electrical passed. Table 1 shows the dielectric properties of the samples. The dielectric properties result was taken from the Vector Network Analyzer (VNA) experiment. By using an open-ended coaxial probe method. The open-ended coaxial probe subsists of an abbreviated part of the transmission line. The magnetic radiation passes along the transmission cable and the reflection takes place when the magnetic radiation encounters an impedance unevenness between the probe and the tissue sample [9]. This measurement gave an impact on the input voltage readings at the receiver circuit.

Table 1.1: The electrical properties of samples[10]

\begin{tabular}{|l|l|l|l|}
\hline Frequency & Samples & $\begin{array}{l}\text { Relative } \\
\text { Permittivity }\end{array}$ & Conductivity \\
\hline \multirow{3}{*}{$10 \mathrm{MHz}$} & $\begin{array}{l}\text { No } \\
\text { agarwood }\end{array}$ & 3.17944 & 0.00850 \\
\cline { 2 - 4 } & A & 2.84180 & 0.00219 \\
\cline { 2 - 4 } & B & 1.60002 & 0.00870 \\
\cline { 2 - 4 } & C & 1.71432 & 0.00204 \\
\hline
\end{tabular}

\section{SYSTEM DESIGN}

\section{A. Coil Sensor}

There are several categories of coils that MIT have been used such as gradiometer, ferrite core coils, and including the solenoid air-core [4],[5]. A hand-wound solenoid air core coil is chosen in this MIT experiment. For this preliminary setup, a couple of set of coils is used to examine and screening the object through the signal captured. The coil is formed by a $1 \mathrm{~mm}$ copper wire winding at Polyvinyl Chloride (PVC). For the transmitter, coil 10 turn coils $(9.1 \mu \mathrm{H})$ winding is used to transmit a $10 \mathrm{MHz}$ while for the receiver used 20 turns coil $(25.9 \mu \mathrm{H})$ winding as in Figure 3. The square box shielding with aluminum foil (see in Figure 4) was applied to the sensors to minimize the impact of noise from the capacitive coupling and other external magnetic fields [4],[6]. The resistor is connected to the impedance matching coils in order to obtain the greatest transfer or to reduce the reflection of the signal from the load [7].

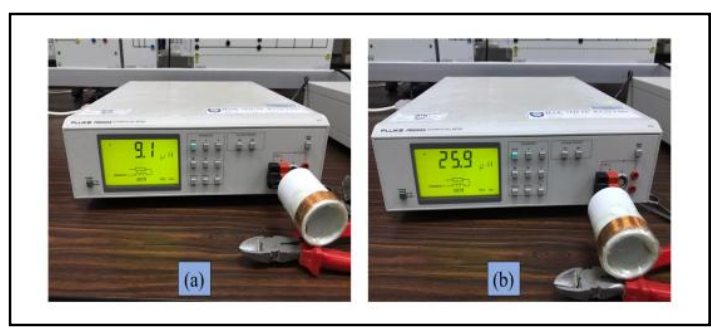

Figure 3: (a) The inductance of the transmitter coil. (b) The inductance of receiver coil.

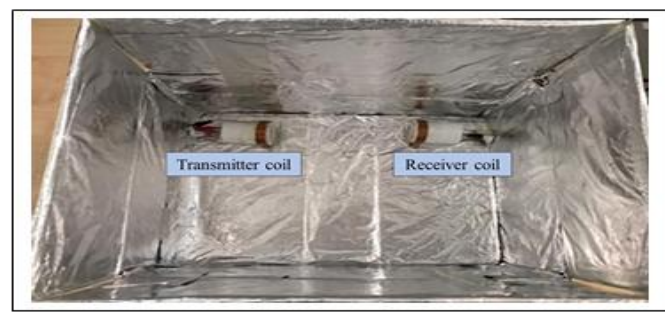

Figure 4: The coil sensors inside the shielding experiment box

\section{B. Transmitter}

Figure 5 shows a simplified schematic of the excitation circuit. AD817 amplifier has a good performance which is a high speed and high output drive capability which able to operate an infinite power amplifier while retaining high signal integrity with a low power supply range. It also has broad unity-gain bandwidth until 50MHz. The $50 \mathrm{MHz}$ unity-gain bandwidth, $350 \mathrm{~V} / \mu$ s slew rate, and $45 \mathrm{~ns}(0.1 \%)$ set-up time make high-speed signal processing possible. If the value of the $\mathrm{R} f$ feedback resistor is zero, the output of the voltage will be exactly equal to one (unity). If the R2 resistor is zero, the voltage gain can approach infinity, but in fact, it is constrained to the open-loop differential gain of the operating amplifiers $\left(\mathrm{A}_{\mathrm{O}}\right)$. In some amplifiers, the open-loop gain can be exceptionally high. The ideal operating amplifier (op-amp) does have a limitless open-loop gain. Adjusting the adjusted feedback resistor $(\mathrm{R})$ to the potentiometer, the circuit is capable of achieving an adjustable gain.

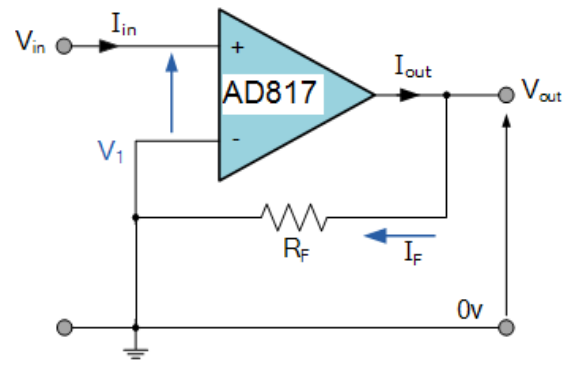

Figure 5: The excitation circuit

\section{Receiver}

The receiver consists of a receiver coil and the AD817 is used as the receiver front end circuit. The signal captured in the receiver coil is then transmitted to the power amplifier for processing. The Block diagram in Figure 6 shows the four stages that the signal undergoes before being passed before sending the signal to the microcontroller for image reconstruction.

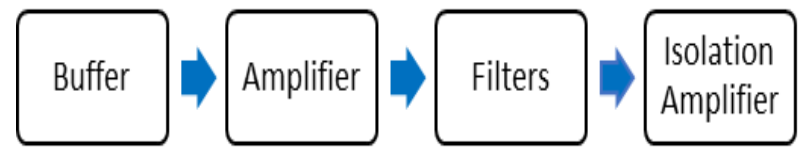

Figure 6: Block diagram for receiving circuit

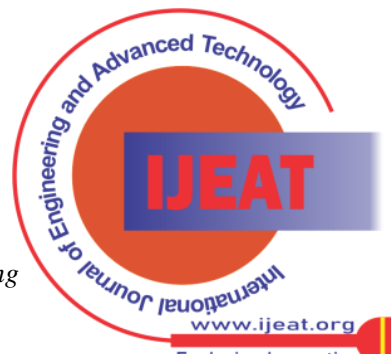


- Buffer

Figure 7 shows the buffer amplifier Provides the transformation of electrical impedance from one circuit to another. The aim of using a buffer is to prevent the signal source from being affected. An HA5002 buffer amplifier is used because it has output current capability is enhanced by a $3 \Omega$ output impedance. Besides, it also offered $1300 \mathrm{~V} / \mu$ s slew rate with $110 \mathrm{MHz}$ of bandwidth. The buffer outcome precisely reflects the input (implying it is within the range of the voltage rails).

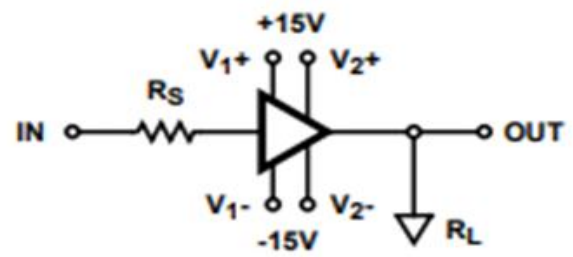

- Amplifier

Figure 7: Buffer amplifier

The amplifier part used AD817 which operates in low power supply either single or dual power source and high-speed op-amp. As shown in Figure 8, a non-inverting amplifier was used. Voltage regulation of the non-inverting operating amplifier is obtained by implementing a small part of the output voltage signal back to the inverting (-) input terminal via the $\mathrm{R} f-\mathrm{R} 2$ voltage divider circuit, which again produces negative feedback.

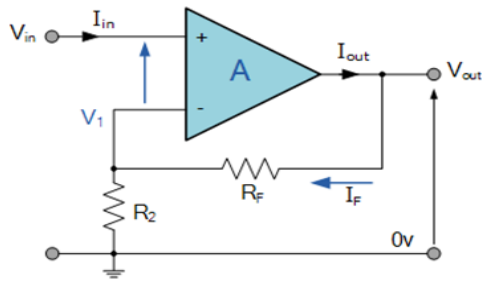

Figure 8: An amplifier circuit using AD817

This non-inverting amplifier circuit with closed-loop configuration produces excellent stability and great high input impedance in the inverting circuit of the amplifier [8]. The voltage gain $(A v)$ can be resolve by Equation (1).

$$
A v=1+\mathrm{R} 1 / \mathrm{Rf}
$$

- Filters

The filter is a circuit able to pass through several frequencies while attenuating other frequencies. As a result, a filter can extract unwanted or worthless frequencies form the useful signal frequencies. For this experiment, a passive bandpass filter was used to isolate or filter predefined frequencies within a particular band or frequency range as in Figure 9. The cut-off frequency or point of a single RC passive filter can be precisely controlled using only a resistor. The higher and lower cut-off frequency points for a bandpass filter can be found using the same formula as in Equation (2).

$$
f c=1 / 2 \pi R c
$$

By cascading the high pass filter with a low pass filter which passed a signal in certain "bandwidth" frequencies is called as bandpass filter. Bandwidth is typically known as the range of frequencies between the two specified frequency cut-off points $(f \mathrm{c})$ can be defined as in Equation (3).

$$
\text { Bandwidth, }(\mathrm{BW})=f H-f L
$$

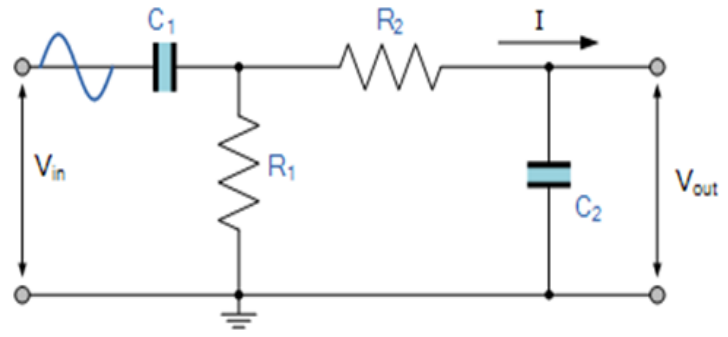

Figure 9: The passive band filter circuit

- Isolation amplifier

An isolation amplifier (also called a unity-gain amplifier) is an op-amp circuit that allows one part of the circuit to be isolated, in order to prevent power from being used, diverted or discarded in one part of the circuit. The intent of the insulation amplifier is to isolate the circuit that occurs before the amplifier from the circuit that tends after it. Figure 10 shows the isolation amplifier circuit connection.

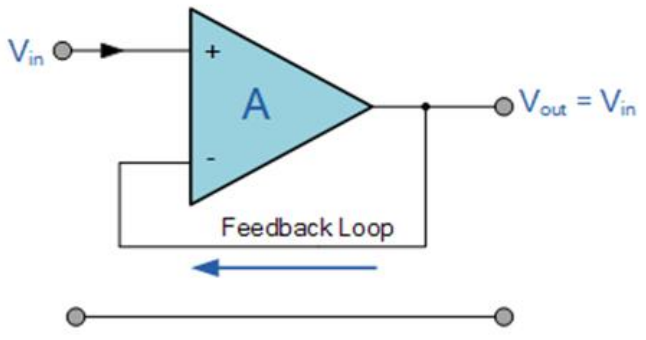

Figure 10: Isolation amplifier circuit

When the output is directly connected to the inverting input so that the responses is $100 \%$. As the Vin is literally equal to Vout handing it a rigid gain of 1 or unit. The input voltage Vin is implemented to the non-inverting input, the amplifier gain is assigned as:

$$
\mathrm{Av}=\text { Vout } / \mathrm{Vin}=+1 \quad \text { Eq. (4) }
$$

\section{EXPERIMENT SETUP}

The experiment is set up (see in Figure 11) focusing to inspect the voltage reaction between transmitter and receiver. It consists of an oscilloscope, power supply, signal generator, experiment box, and transceiver circuit. The experiment boxes in the Figure shown where the samples took place. The experiment box shielding with aluminum foil to prevent the outsource signal disturbance. Power supply generated $\pm 8 \mathrm{v}$ to activated components. Then, the signal generator was set $4 \mathrm{~V}$ peak-to-peak with $10 \mathrm{MHz}$ frequency into the input pin of the transmitter circuit. The transceiver circuit was connected with coil sensors where a channel is used as a transmitter and receiver. The oscilloscope is used to measure the voltage at the input receiver.

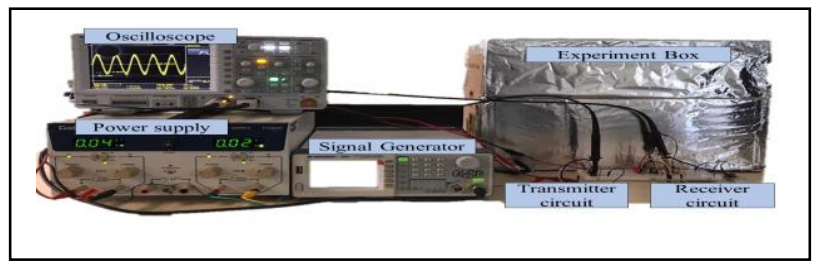

Figure11: The experiment setup

Published By:

Blue Eyes Intelligence Engineering and Sciences Publication 


\section{Exploratory Designing a Magnetic Induction Tomography Sensor Coil Circuit for Agarwood}

\section{RESULTS}

A $10 \mathrm{MHz}$ sinusoidal signal with $4 \mathrm{~V}$ peak-to-peak amplitude was applied to the non-inverting excitation circuit as in Figure 12(a) from the BK PRECISION 4055 signal generator. As the value of the negative terminal is zero the signal was amplified to the maximum voltage is $6.96 \mathrm{~V}$ as in Figure $12 \mathrm{~V}(\mathrm{~b})$.

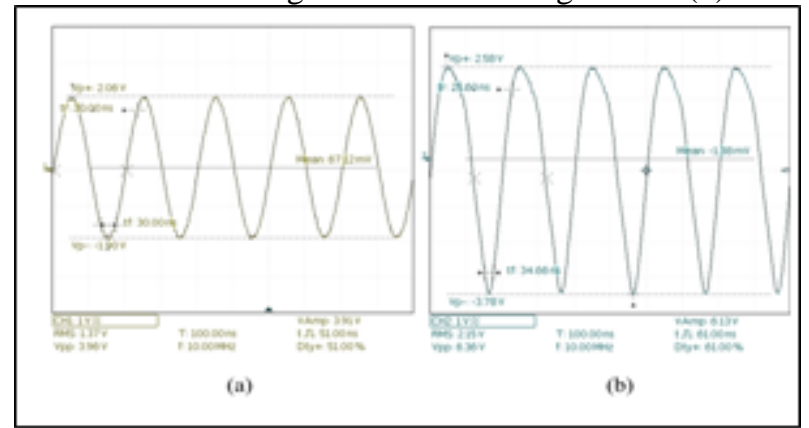

Figure 12: (a)The input signal of the excitation circuit. (b) The output signal of the excitation circuit after amplification

The excitation amplifier amplified the output signal, there has a phase shift between input and output and oscillation occurs when phase shift equals or exceeds 180 degrees (see in Figure 13) at any frequency where the open-loop gain is greater than one[11] achieved by applying $100 \%$ feedback to a high gain amplifier.

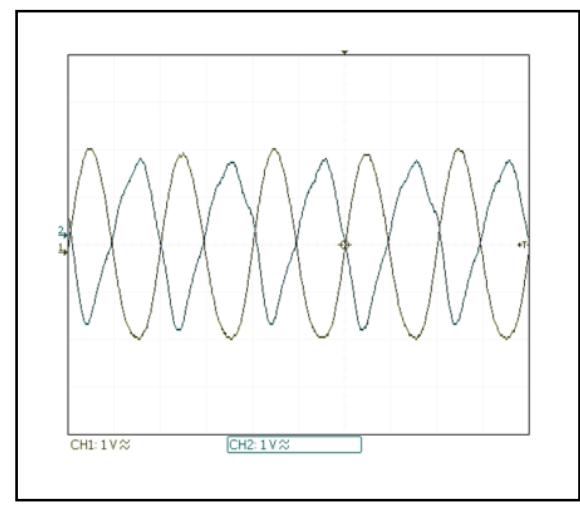

Figure 13: The phase shift occurs between the input signal TX and the input signal RX

After the excitation coil transmitting the signal, the signal going to mix up with the surrounding noise. This can affect the receiver coil to capture the signal. A buffer is connected to avoid signal loss. Figure 14 shows the signal has captured by the input buffer. The voltage is passed constant (Av voltage gain is 1) because the output voltage pursues the input voltage. The signal captured at the receiver is a bit low, 1.12V. This signal was amplified at the next stage.

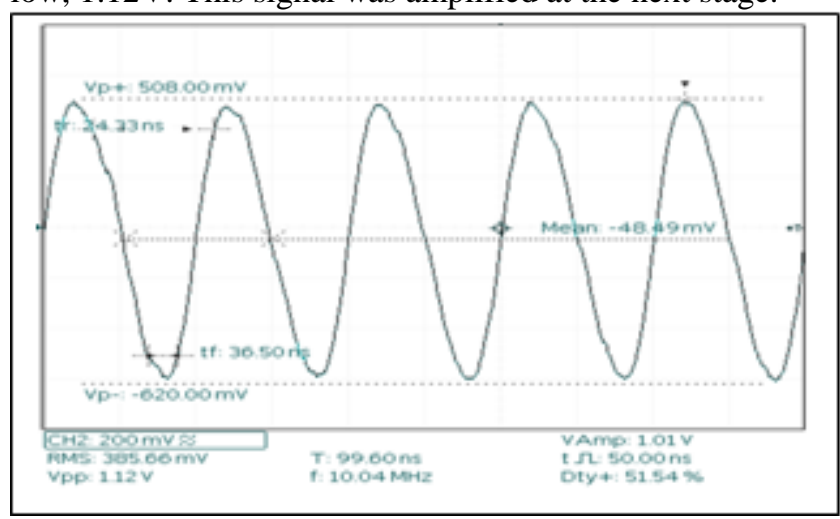

Figure 14: The signal capture by the buffer
Figure 15 shows the output voltage after the signal pass through the amplifier circuit. The output is remains same and not inverted because the input signal is directly connected to the non-inverting pin. So, the output voltage is directly proportional to the input voltage, Vout $=$ Vin. The signal successfully amplified is $3.04 \mathrm{~V}$.

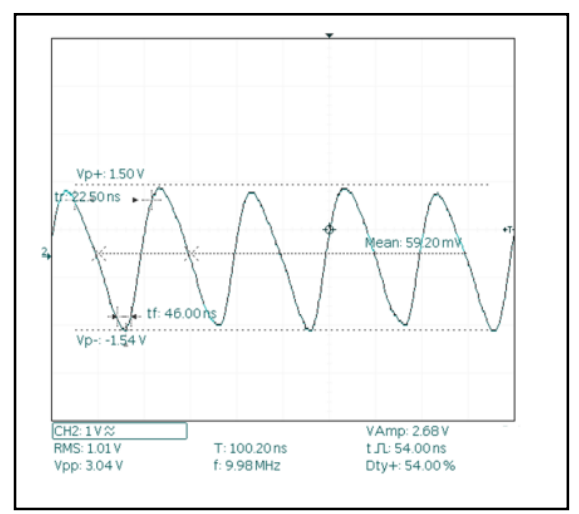

Figure 15: The output signal for the amplifier circuit After the amplification was processed, the signal was taken to the isolation stage. This process made the voltage follower circuit optimal as a unit gain buffer circuit due to its insulation properties. The benefit of the voltage follower is that it could be used when circuit isolation or impedance matching is more significant than amplification since it preserves the signal voltage. This stable signal voltage is important for microcontroller input. The bandpass filter circuit is placed between the amplifier and isolation circuit is to pass frequencies within an assertive range and attenuates frequencies beyond that range. The bandwidth range between $8 \mathrm{MHz}$ until $15 \mathrm{MHz}$. Signals at frequencies outside of the range to which the receiver is tuned could either deplete or degrade the receiver. They can also create unwanted blending substances that crash into the band and conflict with the desired signal. The bandpass filter also improves the signal-to-noise ratio and the efficiency of the receiver.

\section{DISCUSSION}

The fundamental MIT circuit as above has been strongly tested at the agarwood sample to observe the MIT reaction. Figure 16 shows the dry agarwood samples were used in the experiment. The red circle marker is the formation of agarwood in the trunk samples. Each sample has a different size of agarwood formation.

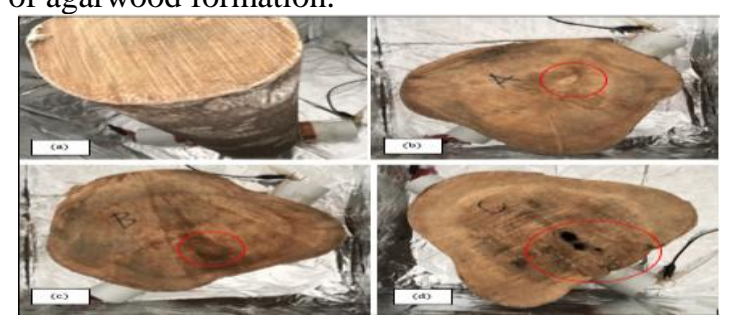

Figure 16: Agarwood samples. (a) sample without agarwood formation, (b) small agarwood formation, (c) moderate agarwood formation, (d) large agarwood formation with a hole

Published By:

Blue Eyes Intelligence Engineering and Sciences Publication 
The receiver captured the voltage response when the voltage was induced at the excitation circuit, the signal was passed through the samples. The input voltage response was recorded at the buffer pin where it is attached to the receiver coil. Then, the input voltage response range without a sample at the receiver was captured is between $1.02 \mathrm{v}$ until $1.58 \mathrm{v}$. The voltage readings are high compared to the input voltage response with samples is low. Table 5.1 shows the best five readings of input and output voltages with samples were taken at the receiver circuit. The input voltage response range for each sample is different. For the sample without agarwood is higher than the samples with agarwood formation. When there is no agarwood formation, the electrical current can pass effortlessly. As the agarwood formation have low conductivity, the electrical is having the challenge to pass. The three samples with agarwood formation show that sample $\mathrm{C}$ has a low input voltage response because sample $\mathrm{C}$ has a large area of agarwood formation. But sample $\mathrm{C}$ also has a hole inside the sample. As the hole or air gave an impact to the voltage readings, the input reading is higher. Compare to sample $\mathrm{B}$, the agarwood formation is bigger than sample $\mathrm{A}$, the reading is lower than $\mathrm{A}$.

Table 4.1: The input and output voltages of samples

\begin{tabular}{|c|c|c|c|}
\hline Samples & $\begin{array}{c}\text { Input voltage } \\
\text { range }\end{array}$ & $\begin{array}{c}\text { Input (V) at } \\
\text { buffer } \\
\text { amplifier }\end{array}$ & $\begin{array}{c}\text { Output (V) } \\
\text { at isolation } \\
\text { amplifier }\end{array}$ \\
\hline \multirow{3}{*}{ agarwood } & $1.02 \mathrm{~V}-1.30 \mathrm{~V}$ & $1.16 \mathrm{~V}$ & $2.88 \mathrm{~V}$ \\
& & $1.10 \mathrm{~V}$ & $2.84 \mathrm{~V}$ \\
& & $1.14 \mathrm{~V}$ & $2.88 \mathrm{~V}$ \\
& & $1.22 \mathrm{~V}$ & $2.96 \mathrm{~V}$ \\
A & \multirow{3}{*}{$976 \mathrm{mV}-1.20 \mathrm{~V}$} & $1.12 \mathrm{~V}$ & $2.92 \mathrm{~V}$ \\
& & $1.14 \mathrm{~V}$ & $2.84 \mathrm{~V}$ \\
& & $1.14 \mathrm{~V}$ & $2.96 \mathrm{~V}$ \\
& & $1.10 \mathrm{~V}$ & $2.92 \mathrm{~V}$ \\
& & $1.00 \mathrm{~V}$ & $2.92 \mathrm{~V}$ \\
\hline \multirow{3}{*}{$\mathrm{B}$} & $940 \mathrm{mV}-1.17 \mathrm{~V}$ & $1.04 \mathrm{~V}$ & $2.80 \mathrm{~V}$ \\
& & $1.00 \mathrm{~V}$ & $2.72 \mathrm{~V}$ \\
& & $1.06 \mathrm{~V}$ & $2.84 \mathrm{~V}$ \\
& & $940 \mathrm{mV}$ & $2.60 \mathrm{~V}$ \\
\hline \multirow{3}{*}{$\mathrm{C}$} & \multirow{3}{*}{$840 \mathrm{mV}-1.18 \mathrm{~V}$} & $920 \mathrm{mV}$ & $2.56 \mathrm{~V}$ \\
& & $1.12 \mathrm{~V}$ & $2.76 \mathrm{~V}$ \\
& & $1.06 \mathrm{~V}$ & $3.00 \mathrm{~V}$ \\
& & $1.06 \mathrm{~V}$ & $2.88 \mathrm{~V}$ \\
& & & $2.84 \mathrm{~V}$ \\
\hline
\end{tabular}

\section{CONCLUSION}

The approach transceiver circuit above were successful and functional for MIT coil sensing. However, receiver circuit sensitivity is hard to achieve. The maximum power-transfer theorem stated that in order to transfer the maximum amount of power from the source to the load, the impedance of the load should match the impedance of the source. Series parallel circuit gives a greater induction factor and therefore more induced voltage for the load of the receiver [12]. The MIT experiment against the agarwood samples shows that input voltage response at the receiver gave a good return at $10 \mathrm{MHz}$. When the dielectric properties are high, the input voltage also high. Diversely, when the dielectric properties are low, the input voltage also is low. As a $10 \mathrm{MHz}$ frequency 306-312, 1999. widely used in medical applications but haven't applied for agriculture application. So, the $10 \mathrm{MHz}$ frequency is selected because the magnetic field has limited power. Power increases at low frequencies are strongly dependent on the parameters of the coil. In the case of a multi-turn coil, this output power is reduced due to skin and proximity effects [13].

\section{ACKNOWLEDGMENT}

This research was supported by Ministry of Higher Education (MOHE) through Fundamental Research Grant Scheme (FRGS/1/2018/TK04/UTHM/02/28) or Vot No. K087. The hors also would like to dedicate special thanks to Research Management Centre (RMC) of Universiti Tun Hussein Onn Malaysia for its support to achieve the goal of research work.

\section{REFERENCES}

1. A. J. Peyton et al., "Development of Electromagnetic Tomography (EMT) for Industrial Applications. Part 1: Sensor Design and Instrumentation," 1st World Congr. Ind. Process Tomogr., vol. 3, pp.

2. M. S. B. Mansor et al., "Magnetic induction tomography: A brief review," J. Teknol., vol. 73, no. 3, pp. 91-95, 2015.

3. D. Gürsoy and H. Scharfetter, "Optimum receiver array design for magnetic induction tomography," IEEE Trans. Biomed. Eng., vol. 56, no. 5, pp. 1435-1441, 2009.

4. A. Stubendekova and L. Janousek, "Impact of defect extent on swept frequency eddy current responses in non-destructive evaluation," Electr. Eng., vol. 99, no. 4, pp. 1275-1281, 2017.

5. J. García-Martín, J. Gómez-Gil, and E. Vázquez-Sánchez, "Non-destructive techniques based on eddy current testing," Sensors, vol. 11, no. 3, pp. 2525-2565, 2011.

6. S. Watson, R. J. Williams, H. Griffiths, W. Gough, and A. Morris, “A transceiver for direct phase measurement magnetic induction tomography," Annu. Reports Res. React. Institute, Kyoto Univ., vol. 4 pp. 3182-3184, 2001

7. L. Ma and M. Soleimani, "Magnetic induction tomography methods and applications: A review,” Meas. Sci. Technol., vol. 28, no. 7, 2017.

8. [8] A. J. Peyton, Electromagnetic induction tomography. Elsevier Ltd, 2015.

9. A. La Gioia et al., "Open-Ended Coaxial Probe Technique for Dielectric Measurement of Biological Tissues: Challenges and Common Practices," Diagnostics, vol. 8, no. 2, p. 40, 2018.

10. N. Ishak, C. K. Lee, S. Zarina, and M. Muji, "Dielectric properties measurement of Agarwood using Vector Network Analyzer for frequency $200 \mathrm{MHz}$ until $1 \mathrm{GHz}$," in 2nd International Conference on Computer Science, Electrical \& Electronic Engineering 2020, 2020, pp. 1-11.

11. M. Al-Hawari, A. I. Aulia, A. Rudin, Rohmadi, I. Muttakin, and W. P. Taruno, "Receiver Circuit Design for Signal Conditioning in Magnetic Induction Tomography System," Proc. 2017 5th Int. Conf Instrumentation, Commun. Inf. Technol. Biomed. Eng. ICICI-BME 2017, no. November, pp. 230-235, 2018.

J. I. Agbinya, "Performance of magnetic induction communication systems using induction factors," Wirel. Pers. Commun., vol. 70, no. 2 pp. 945-968, 2013.

13. Morag and Levron, "Output Power Limit in Energy Harvesting Systems Based on Magnetic Induction Incorporating High-Frequency Effects," Instruments, vol. 3, no. 2, p. 26, 2019. 


\section{AUTHORS PROFILE}

Nurfarahin Ishak was born on 1990 in Perak, Malaysia. She received the Diploma (Electronic Computer Engineering) from Ungku Omar Polytechnic in 2011 and B. Sc. (Electronic Engineering) in 2016 from Universiti Tun Hussein Onn Malaysia. Currently, she is doing M. Sc (Electrical Engineering) at Universiti Tun Hussein

Onn Malaysia.

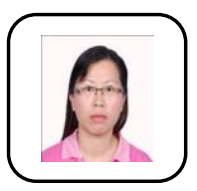

King Lee Chua was born in Sarawak, Malaysia, in 1976. She received the Diploma in Electronic Engineering from the Polytechnic Kuching, Sarawak, Malaysia, in 1997, the Bachelor of Science in Electrical Engineering (Control and Instrumentation) from the Universiti Teknologi Malaysia, Johor, Malaysia, in 2002, the Master of Science in the Universiti Kebangsaan Malaysia, Selangor, Malaysia, in 2005, and the Doctor of Philosophy in Electric Engineering from the Universiti Tun Hussein Onn Malaysia, Johor, Malaysia, in 2015. She has been engaged in research activities with industry in the field of electromagnetic integrity on chip level using GTEM cell and anechoic chamber, other electromagnetic compatibility-related topics. Her research interests include FPGA design, embedded system design and applications, and chip level radiated emission-related topics.

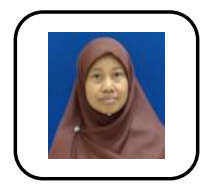

Siti Zarina Mohd Muji was born in Batu Pahat, Malaysia in 1978. She received B. Sc. (Electrical and Electronic Enginnering (Computer)) And M. Sc (Electrical and Electronic Engineering (Data Communication)) from Universiti Sains Malaysia in 2001 and 2004 respectively and the Ph.D. (Electrical Engineering (Electronic Instrumentation)) from Universiti Teknologi Malaysia in 2012. She is a member of the professional membership Board of Engineer (BEM) since 2014. Her research interest is Embedded system and Tomography.

Abdul Azlin Bin Abdul Latip was born in Balik

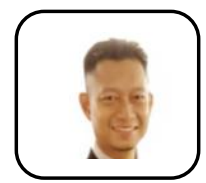
Pulau, Penang Malaysia in 1981. He received a Diploma in Electronic Communications in 2002 from Polytechnic Jitra, Kedah, and received B. Sc. Management (minor politic \& major marketing) from Malaysia Sains of University in 2015. Experienced working and involved in RF and Digitalize at Keysight Technologies (M) Sdn. Bhd. Currently, he is involved in data science and analysis at Smart Modular Technologies (M) Sdn. Bhd.

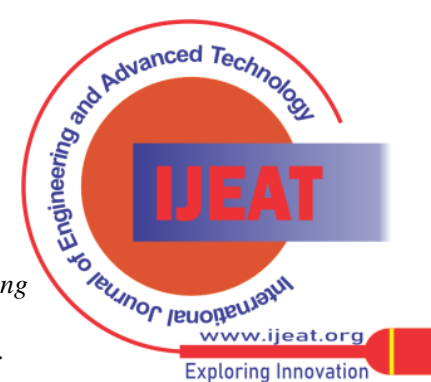

